\title{
Signals Are Minimal CaUSes
}

Marc Artiga ${ }^{1}$

(forthcoming in Synthese)

\begin{abstract}
Although the definition of 'signal' has been controversial for some time within the life sciences, current approaches seem to be converging toward a common analysis. This powerful framework can satisfactorily accommodate many cases of signaling and captures some of its main features. This paper argues, however, that there is a central feature of signals that so far has been largely overlooked: its special causal role. More precisely, I argue that a distinctive feature of signals is that they are minimal causes. I explain this notion, suggest some strategies for identifying its instances and defend its relevance by means of conceptual and empirical considerations.
\end{abstract}

\section{Introduction}

The natural world is full of signals. Meerkats perform alarm calls to warn their fellows about approaching predators, female fireflies emit nocturnal light signals with their lower abdomens to attract males, and gazelles stot to inform predators that they have been spotted. Indeed, the idea that microorganisms and even plants are able to communicate, not only with organisms of the same species, but also across biological realms, is progressively gaining prominence. Signaling might be as widespread as life itself.

In biology and philosophy there is a growing interest in identifying the key elements involved in this striking phenomenon. As a result of this effort, a partial agreement on the features that are required for a state to qualify as a signal seems to be approaching. The resulting view undoubtedly captures some of signaling's central aspects, but I will show that this analysis is still incomplete. More precisely, I will argue that a careful look at the evidence gathered by scientists, together with

\footnotetext{
${ }^{1}$ marc.artiga@uv.es. Departament de Filosofia, Facultat de Filosofia i C.de l'Educació, Universitat València, Av. Blasco Ibàñez 30, 46010 Valencia (Spain)
} 
conceptual considerations, suggests that signals play a special causal role, that is, they are what I will call 'minimal causes'. I will spell out the notion of a 'minimal cause' and argue that this is a suitable additional condition for a state to qualify as a signal. Finally, I will argue that this approach provides an original and illuminating perspective on some classical issues, such as the debate between informationalists and manipulationists and the arbitrariness of signals.

The paper is organized into four main sections. In section 2 I will briefly set out the most common view of animal signaling, which I take to be a version of the socalled 'teleological theory'. The next section argues that this account is probably incomplete, and I will motivate this view by discussing some examples. In section 4 I will spell out and defend the idea that signals are special sorts of causes and that this feature should be included in their definition. Finally, I will explore the connections between the idea of a minimal cause and other debates in the literature on animal communication, and will end with some conclusions.

\section{The Received View}

A classical definition of a signal is provided by Maynard-Smith and Harper (2003: 3):

[A signal is] any act or structure that alters the behavior of other organisms, which evolved because of that effect, and which is effective because the receiver's response has also evolved.

This condensed statement provides some of the key elements that are widely thought to constitute the essential features of signaling (Allen and Bekoff, 1997; Bradbury and Vehrencamp, 1998: 22; Diggle et al. 2007: 3; Hasson, 1994; Otte, 1974; Searcy and Novicky, 2005; Scott-Phillips, 2008; Shettleworth, 2010: 512; Wiley, 2013: 120; Zahavi and Zahavi, 1997). First, it asserts that signals involve various mechanisms (often within different organisms), some producing structures or acting in certain ways (the senders), and others interpreting them (the receivers). ${ }^{2}$ Secondly, the act or structure has evolved in order to have this effect on the interpreter, that is, the structure has that effect as its evolutionary function. 3 Thirdly, the organisms receiving the signal must have an evolved

2 In most of the examples we will discuss, the roles of sender and receiver are played by different organisms, but they can also be parts of the same organism (e.g. brain states, hormones).

3 Probably, processes other than natural selection (such as learning) can also ground functions (Abrams, 2005; Frick et al., 2019; Shea, 2018). Nonetheless, for simplicity, and given that most 
response, that is, they must have the function of producing a certain behavior when the act or structure obtains. In short, signals are intermediate states (acts or structures) that lie between a sender and a receiver, in which both agents have certain evolutionary functions. The main features of this view are represented in figure 1:

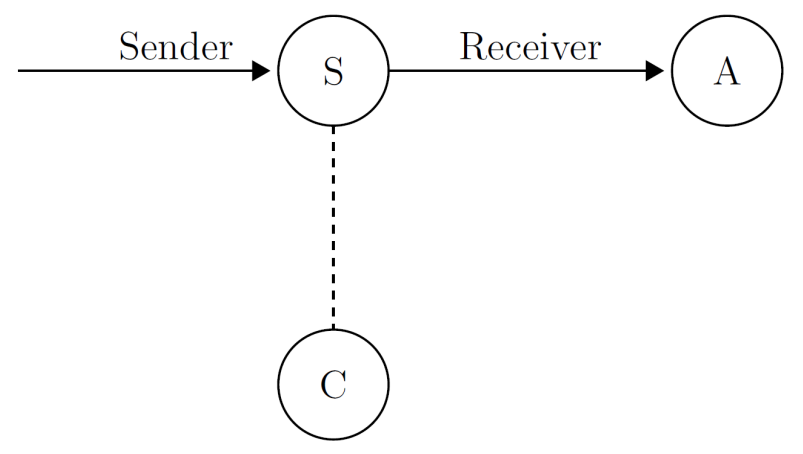

Figure 1: The Received View. 'S' stands for the act or structure (signal), 'C' for the content or condition represented, and 'A' for the act of the receiver. Arrows stand for causal relations and dashed lines for representational relations.

The key elements of this framework can be used, for instance, to distinguish signals from cues, coercion and camouflage. A cue is a state or structure that produces an evolved response from the receiver, but which has not evolved because of those effects (i.e. a proper sender is missing). In contrast, coercion and camouflage involve situations in which the receiver has not evolved to react to the signal (Scott-Phillips, 2008: 389). Below I will present other examples that seem to suggest that this approach is able to rightly distinguish and accommodate many other phenomena. 4

For the purposes of this paper, it is important to highlight two other features that are not explicitly stated in the previous definition, but which are often assumed to be central as well. They are mentioned, for example, in Bradbury and Vehrencamp's (1998: 356) analysis:

of the examples we will discuss involve evolutionary functions, I will focus on those.

4 Nonetheless, not everyone agrees with this picture. For instance, some people hold that an evolved sender is not required (e.g. Stegmann, 2009; cf. Artiga, 2014). Fortunately, I think that nothing I'll say here depends on this claim. Others, however, oppose the idea that animal communication involves an exchange of meaningful signals (Dawkins and Krebs, 1978; Rendall et al. 2009; Rendall and Owren, 2013). This latter approach will be briefly discussed in section 4.3 . 
We shall define a signal to be any action or trait generated by one animal (the sender) which provides information used by another animal (the receiver) to select an action beneficial to both parties. [emphasis added]

Two elements of this analysis are worth emphasizing. First, many authors assume that senders and receivers must have, at least, partial common interest (GodfreySmith, 2013). The rationale behind this idea is that if the sender did not benefit on average from engaging in this activity, it would not produce signals, and if the receiver didn't obtain some benefit, it would stop listening (cf. Godfrey-Smith and Martinez, 2013). Secondly, signals are states upon which receivers condition their actions. Thus, there must be a range of acts or structures (at least two), such that receivers select their behavior according to the presence of one of them (Wiley, 1994: 163; Scott-Phillips, 2008: 388). In other words, there have to be different signals (e.g. an alarm call and its absence), which are supposed to generate different behaviors and are associated with different success conditions.

As an illustrative example, consider the firefly's flashing: there is a sender (a female firefly), a range of intermediate states (flashing with a specific pattern, no flashing) and a receiver (a male firefly). Furthermore, the sender has the function of emitting light in certain circumstances (when the female is willing to mate) and not producing it when this condition is absent, and the receiver is supposed to act in certain ways when the light is sent. Consequently, this view entails that $\mathrm{S}$ is a signal that plausibly means something like there is a female willing to mate. In philosophy, this approach is usually called the 'teleological' or 'teleosemantic' theory, because the notion of function plays a key role (Neander, 2012; Papineau and McDonald, 2006). In the remainder of this paper, I will refer to this account as the 'Received View', or 'RV' for short.

\section{Assessing the Received View}

Despite the fact that RV is relatively successful in identifying genuine cases of signals in many domains, I think there is a central feature of signals that it fails to capture. One way of motivating this suggestion is to consider some counterexamples. Sterelny (1995: 256), for instance, argues that saliva, which no biologist would categorize as a signal, 5 might meet these conditions:

5 In certain special circumstances, saliva might actually work as a signal. For instance, some 
It will turn out that saliva represents food. The production of saliva in the mouth adapts the digestion system to the reception of food. So it mediates between our mechanisms of ingestion and digestion: our digestive systems can only fulfil their function normally if saliva is correlated with food. Yet surely this is a nonrepresentational mechanism. I think the relations between many merely physiological mechanisms will meet Millikan's condition. ${ }^{6}$

This passage suggests that saliva seems to instantiate all the characteristics that $\mathrm{RV}$ requires for a state to qualify as a genuine signal: the salivary glands (the sender) have the evolutionary function of delivering saliva (the signal) to the mouth and stomach (the receiver) when there is food around (C), and the success of the digestive process seems to partly depend on there being some food. Thus, saliva seems to qualify as a signal of food according to RV.7

Sterelny's counterexample concerns intraorganismic processing, but similar examples can be found in organism-to-organism communication. Evidence gathered over recent decades, for example, has convinced many biologists that plants communicate with each other through a number of processes (Baluska et al. 2006; Ueda et al., 2012). When plants are exposed to stress factors such as disease, extreme heat, injury or herbivory, many of them release volatile organic compounds (VOCs), which are transported by wind and activate in other plants a range of physiological adjustments, in response to or in preparation for those situations (Baluska and Ninkovic, 2010). For instance, tomato plants (Solanum lycopersicum) respond to herbivory by producing an 18-amino acid hormone called 'systemin' that induces the activation of over 20 genes that together regulate a defensive response (Ryan, 2000; Corrado et al. 2007). Systemin is released, distributed by wind, and binds to a pair of receptors (SYR1 and SYR2) that induce an elevated transcription of factors in unchallenged plants that makes them more attractive to parasitoids, more resistant to pests, and causes an increased response to wounding (Coppola et al., 2017; Wang et al. 2018).

stingless bees use saliva to lay a trail that indicates that an important source of food has been found (Schorkopf et al. 2007). Nonetheless, it is much harder to accept that saliva plays a representational role simply in virtue of how it is used in digestion.

6 Millikan (1984) was one of the first philosophers to propose a teleological theory of representations along the lines of RV.

7 As a reviewer suggested, one might object that it is not obvious that the mouth or the stomach has the function of responding to the presence of saliva. In any case, I think this example is not only of historical interest, but also points in the direction of a range of similar examples that do seem to raise a real challenge. 
Compare this process to pollination. Anemophily is a form of pollination whereby pollen is distributed by wind. Almost all gymnosperms are anemophilious, as well as at least $10 \%$ of angiosperms such as oaks, grasses and shrubs (Ackerman, 2000; Friedman and Barrett, 2009). Flowers usually emerge early in the spring and possess large stamens and feathery stigmata exposed to wind currents. Anemophilous pollen grains also tend to be smaller and lighter than pollen grains from entomophilous plants (i.e. those pollinated by means of insects) and have very little nutritious value for animals. Thus, they have been designed by evolution to be produced at the right time (spring) and facilitate air transport.

Now, both VOCs and pollen seem to satisfy RV. There are senders that have the function of emitting these structures, which are carried by wind. They also trigger a range of processes in the receiving plant, which certainly have evolutionary functions. Furthermore, these structures are sent in particular circumstances, which are important for the success of the response: VOCs are produced in response to stressful events and induce better preparation for them. In pollination, this is triggered by stimuli linked to the onset of spring (daylight length, etc.), when conditions are more favorable for the success of germination. Thus, according to RV, both should be classified as signals, but they are obviously not: pollination is not a process of communication, whereas the diffusion of VOCs is usually classified as such. Thus, there must be some difference here that is relevant for distinguishing signals from non-signals.

Tool teaching in chimpanzees provides a different kind of example. The use of tools by chimpanzees is transmitted through social learning and varies between populations, so it is usually considered a form of culture. For example, some populations of chimpanzees insert wooden sticks into termite mounds to 'fish' them. Sometimes while fishing, female chimpanzees transfer their sticks to their offspring in order for the youngster to learn how to use them (Musgrave et al. 2016). Also in this case there seems to be a sender (the mother chimp), a receiver (the offspring) and something transmitted (the stick), which is supposed to produce a behavior (termite fishing) in certain circumstances (in front of a termite mound). Furthermore, the presence of a termite mound seems to be the success condition of stick-delivery, so according to RV, tool transfer should count as a signal of the presence of a termite nest, which again is the wrong result. In particular, this way of classifying behaviors would conflate different kinds of 
teaching that should be distinguished.

Similar concerns have been raised by others (Kalkman, 2019; Scarantino, 2013a, 2013b). ${ }^{8}$ These considerations suggest that there might be an important feature of signals that may have escaped our attention. Saliva, pollination and tool transfer seem to satisfy the conditions that are usually assumed to define signals, but they are not classified as such. Consequently, RV probably fails to capture an important feature of signals. Furthermore, these examples are interesting because of the existence of relevantly similar processes (the release of VOCs in plants and alarm calls in chimpanzees) that actually count as genuine instances of communication. Thus, careful reflection on these cases might reveal what is missing in RV. In what follows, I will argue that RV should be supplemented with the idea that signals are minimal causes.

\section{Signals as minimal causes}

Let us think again about plant communication. What is the difference between VOCs and pollen that leads us to classify the former but not the latter as a signal?

The idea I would like to explore is that signals have a distinctive causal profile. Much attention has been devoted to the causal relevance of semantic information, but I think the distinctive causal contribution of the vehicle has been largely overlooked. First, notice that signals are not epiphenomenal or causally inert; they do make an important difference with respect to behavior. However, in some sense, their causal contribution is minimal. Note, for instance, that in describing the effects of signals, scientists typically use expressions such as 'induce', 'activate', 'release' and 'trigger'. These verbs suggest that the primary role of paradigmatic examples of signals is to initiate the appropriate behavior in the right circumstances. Thus, the kind of benefit that signals produce does not primarily depend on the properties of the vehicle, but rather on the properties of the thing

8 Nonetheless, I think that some objections along the same lines actually fail to challenge RV (Scarantino, 2013a: 76; Scott-Phillips, 2008). RV, for instance, rightly excludes reciprocal interactions from being signals. Consider grooming: it alters the behavior of another individual (toward reciprocity) and probably evolved because of this effect, but in this case there is no clear condition $\mathrm{C}$ that grooming is supposed to correspond to, and whose presence causes the recipient's action to increase fitness. Certainly, sometimes grooming works as a signal, as when a chimpanzee engages in this activity in order to indicate her willingness to build an enduring relationship (de Waal, 1982). However, in this case, the sender's positive attitude toward engaging in a relationship plays the role of $\mathrm{C}$, so RV correctly entails that it is a signal (cf. Kalkman, 2019). 
represented. In the case of genuine signals, the medium is not the message. This is the key intuition I will try to capture with the notion of a minimal cause.

More precisely, according to what I will call 'Minimalism', a necessary condition for a state $\mathrm{S}$ to qualify as a signal is that it is a minimal cause:

MiNiMALISM: $\mathrm{S}$ is a signal only if $\mathrm{S}$ is a minimal cause of behavior.

The key intuition behind Minimalism is that in paradigm cases of signaling the intrinsic properties of the state fail to explain behavior. This idea connects with a remark attributed to J. B. S. Haldane, to the effect that 'a general property of communication is the pronounced energetic efficiency of signaling: a small effort put into the signal typically elicits an energetically greater response' (Wilson, 1975: 176). Likewise, it is inspired by Wiley's (1994: 162) definition of signals as 'any pattern of energy or matter produced by one individual (the signaler) and altering some property of another (the receiver) without providing the power to produce the entire response' [emphasis in the original].

Minimalism holds that signals are minimal causes. Unfortunately, the notion of a 'minimal cause' is hard to define in detail. There are various criteria for distinguishing causes, but no standard test for ascertaining minimal causes. For this reason, I will try to illuminate this notion by providing two necessary conditions for a state to qualify as a minimal cause (whether they might also count as sufficient conditions will be discussed below). As a first approximation:

NEC: $S$ is a minimal cause of behavior A only if:

(1) $S$ is a cause of $A$.

(2) $\mathrm{S}$ is not an enabling cause of A.9

The goal of this section is to spell out these two claims and show how they can help to deal with the liberality problem faced by RV. Before going into details, however, an important clarificatory point is in order. Recall that on the Received View, a signaling system is composed of mechanisms that have been designed by evolution (or by some other stabilizing process; see footnote 2). In accordance with this idea, what is relevant for being a minimal cause is whether (1) and (2) held in the design

9 These two conditions could be expressed more succinctly as follows: ' $\mathrm{S}$ is a non-enabling cause of A.' Nonetheless, for clarity of exposition, I prefer to keep these two claims separate here. 
conditions that explain the existence and stabilization of the signaling mechanisms. In other words, what is crucial is whether $\mathrm{S}$ was a non-enabling cause in evolutionary normal conditions, not whether these conditions are currently satisfied (although, of course, the latter is usually used to inform us about the former). This point will be important later on.

In what follows, I will try to explain conditions (1) and (2) in more detail and show how they can be used to address the previous counterexamples. Furthermore, I will show how these two claims connect with empirical research on animal signaling.

\subsection{Causing Behavior}

Claim (1) asserts that signals are causes of behavior. To explain and justify this property, I will rely on Woodward's (2003) widely employed interventionist account. Woodward analyzes causal relations by appealing to certain counterfactuals and interventions. ${ }^{10}$ More precisely, according to him:

(CAUSE) $\mathrm{X}$ causes $\mathrm{Y}$ if and only if there are background circumstances $\mathrm{B}$ such that if some (single) intervention that changes the value of $\mathrm{X}$ (and no other variable) were to occur in $\mathrm{B}$, then $\mathrm{Y}$ or the probability distribution of $\mathrm{Y}$ would change. (Woodward, 2010: 290)

In other words, $\mathrm{X}$ is a cause of $\mathrm{Y}$ iff, having fixed all other variables besides $\mathrm{X}$ and $\mathrm{Y}$ to a certain value, there is a possible intervention on $\mathrm{X}$ that will change $\mathrm{Y}$. As an illustrative example, consider the causal relationship that holds between striking a match, the presence of oxygen, and fire. In the causal graph depicted in Figure 2, the match is represented with a variable $\mathrm{X}$, which can take two values \{strike, nostrike\}, oxygen is represented with a variable $\mathrm{O}$, which can take two values \{present, not-present\} and fire is represented with a variable $\mathrm{Y}$, which can also take two values \{occurring, non-occurring\}. Striking the match causes fire because changing the variable of $\mathrm{X}$ from no-strike to strike would produce a change in $\mathrm{Y}$ (from non-occurrent to occurrent fire), given that the other variables are set to certain values (e.g. oxygen is present).

\footnotetext{
10 'Intervention' is here a technical term: it roughly means an ideal manipulation by which we change the value of a particular variable.
} 


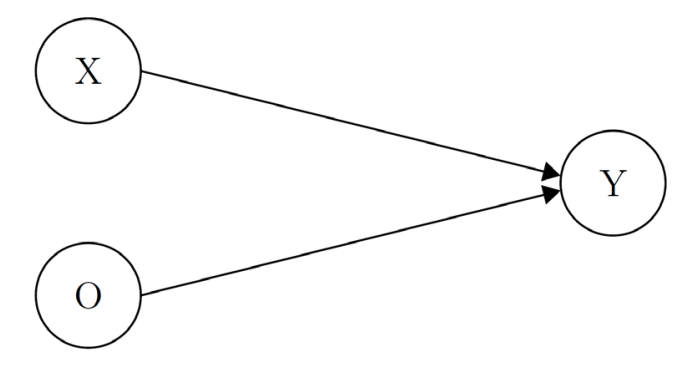

Figure 2: Modeling the relationship between striking a match and fire.

To use the interventionist framework in the context of our discussion, we just need to assume that Figure 1 represents a causal graph (see also Calcott et al., forthcoming) and interpret state (S), the referent (C) and the receiver's action (A) as variables. Each variable can take two values $\left\{S_{0}=\right.$ signal absent, $S_{1}=$ signal present $\},\left\{\mathrm{C}_{0}=\right.$ referent absent, $\mathrm{C}_{1}=$ referent present $\}$ and $\left\{\mathrm{A}_{\mathrm{o}}=\right.$ no behavior, $\mathrm{A}_{1}=$ behavior\}. For each variable, let us stipulate that subscript 1 represents the situation in evolutionary normal conditions, that is, in the conditions that account for the selection of the sender-receiver structure (Millikan, 1984: 33). So $\left\{S_{1}, C_{1}\right.$, $\left.A_{1}\right\}$ represents a scenario in which there is the signal, the referent and the right behavior.

Now, according to CAUSE, $\mathrm{S}$ causes behavior A iff there are background circumstances such that if some (single) intervention that changes the value of $S$ were to occur in $\mathrm{B}$, then $\mathrm{A}$ or the probability distribution of $\mathrm{A}$ would change. Consider a particular case where the initial conditions consist of the absences of the signal, the represented feature and the behavior (i. e. $<S_{o}, C_{0}, A_{0}>$ ). If, in this situation, manipulating the signal variable (from $S_{o}$ to $S_{1}$ ), and only this one, leads to a change in the action (from $\mathrm{A}_{0}$ to $\mathrm{A}_{1}$ ), then the signal is a cause of behavior.

Interestingly, this is not just a conceptual point. When scientists are actively trying to identify signals in the wild, this is one of the main strategies they pursue. Consider, for instance, playback experiments. In a famous experiment, Seyfarth et al. (1980) recorded the call that monkeys performed when they detected a predator, and played it in the absence of predators (i.e., in $\left.<\mathrm{S}_{0} \mathrm{C}_{0}, \mathrm{~A}_{0}\right\rangle$, they changed the value of $S$ to $S_{1}$ to see whether $A$ changed from $A_{0}$ to $A_{1}$ ). The fact that in these circumstances the signal sufficed to elicit the behavior, was taken to provide substantial evidence for the claim that it was indeed a signal. Playback experiments remain one of the key paradigms for studying animal communication 
(Cheney and Seyfarth, 2007) and have been used, for instance, in Diana monkeys (Zuberbühler, 1999), meerkats (Manser et al., 2001), Campbell's monkeys (Zuberbühler, 2000), marmosets (Rogers et al., 2018), and chickens (Evans and Evans, 1999), among many others. This idea is also closely related to Macedonia and Evans's (1993) suggestion that one of the criteria for distinguishing signals (what they call the 'perception criterion') is that in the absence of the eliciting stimulus, the signal should suffice for receivers to select appropriate responses. ${ }^{11}$

Two points are worth stressing. First, to satisfy this condition, it is not enough for the signal to suffice for triggering $a$ behavior. It has to generate the behavior that in normal conditions would be the right one, given evolutionary normal circumstances. For instance, Evans and Evans (1999) compared the behavior caused by some calls of male chickens (Gallus gallus domestiucs) to other kinds of responses, and only when they found that those calls trigger a behavior that is adequate for food did the authors conclude that these are indeed food calls (see also Evans and Evans, 2007; Seyfarth and Cheney, 2017). This feature is also implicit in our model: we stipulated that ' $A_{1}$ ' refers to the response given to $S_{1}$ in normal evolutionary conditions, i.e. in those circumstances that positively account for the selection of the mechanism. As a result, by assuming that the signal triggers $A_{1}$, we assume that the signal should suffice for triggering the behavior whose success condition is $\mathrm{C}_{1}$.

The second caveat is that playback experiments that follow this simple scheme might not work for all cases. In particular, it has recently been pointed out by some scholars that some signals are context-sensitive, in the sense that $\mathrm{S}$ only leads to action if another contextual feature is present (Wheeler and Fisher, 2012, 2015; Scarantino and Clay, 2015; see also Smith, 1991). The rooster's production of alarm calls, for instance, is sensitive to subtle differences in the nature of the audience (Karakashian et al. 1988). In these cases, $S_{1}$ might not suffice for eliciting the response. Nonetheless, it is still true that signals cause behavior: there are certain

11 Another criterion provided by Macedonia and Evans (1993: 179), which is still very important in actual research (Shettleworth, 2010: 515) is production specificity: a signal that refers to a particular object must reliably be given in its presence, and not under other conditions (see also Smith, 1991: 215). Although this criterion might be heuristically useful for identifying signals, I presume it probably fails to point to a distinctive property of signals. In short, my worry is that if this criterion is understood strongly, then it is incompatible with the possibility of misrepresentation (i.e. the fact that signals are sometimes given in the wrong conditions; see Scarantino, 2013b), whereas weakly understood it just claims that signals carry correlational information of whatever they represent, which probably follows from RV above, and does not seem to distinguish signals from other causes. 
background circumstances (which might include contextual features) in which intervening on the presence of the signal actually makes a difference concerning the production of the behavior. Context-dependence just implies that we might need to intervene on other variables besides $\mathrm{S}, \mathrm{C}$ and $\mathrm{A}$ in order to ascertain whether a particular act or structure satisfies the condition (1) for being a minimal cause.

\subsection{Not an enabling cause}

Let us now consider condition (2) of the proposal, which asserts that signals are not enabling causes of $\mathrm{A}$. This is a central aspect of the approach, since it will decisively contribute to accommodating the previous counterexamples, but it is also harder to specify.

First of all, let us define what it is for a receiver to be able to F. Here I'm using the word 'able' in the same sense in which we say that robin birds are able to sing, bacteria are able to divide and plants are able to perform photosynthesis. Unfortunately, none of the main approaches that have been developed for analyzing the notion of 'ability' seem to be adequate for capturing the sense intended here. In philosophy, the most prominent account analyzes abilities in terms of conditionals that relate to the agents' desires, beliefs and intentions, whereas in logic and linguistics this notion is interpreted in terms of restricted possibilities (Maier, 2014). The first kind of approach is ill-suited for organisms such as bacteria or plants, and the second one is too liberal and is probably inadequate for drawing the key distinction between enabling and non-enabling causes.

One suggestion I would like to explore for our purposes is to analyze 'ability' in terms of mechanisms. More precisely, I will assume that $\mathrm{M}$ is able to $\mathrm{F}$ iff $\mathrm{M}$ contains a (complete or nearly complete) mechanism for F-ing. 'Mechanism' should be understood here in the sense employed in the recent mechanistic literature (Machamer et al. 2000; Glennan, 2002). A mechanism for a phenomenon 'consists of entities (or parts) whose activities and interactions are organized in such a way that they produce the phenomenon' (Glennan, 2017: ch. 2). Indeed, we rely here on the 'functional sense of mechanism' (Garson, 2013), since the relevant mechanisms must have been designed by evolution or some other 
stabilizing process to have this effect. Robin birds, for instance, are able to sing because they possesses the required entities and activities (lungs, syrinx, ...), which are organized in such a way that songs are produced. Likewise, bacteria are able to divide because they possess the required mechanism for binary fission. Consequently, 'being able' comes in degrees: all things being equal, the more parts and activities an entity lacks for performing F, the less able it is to F. Moreover, some parts and activities might be more important than others.

This notion of ability can be used to define an 'enabling cause': an entity is an enabling cause of $\mathrm{F}$ iff it makes something able to $\mathrm{F}$. For instance, a plant cell that lacks a chloroplast is unable to perform photosynthesis, in the sense that it lacks some of the key components that constitute the mechanism for photosynthesis. If a biologist inserts a chloroplast into this plant cell, then this action should be classified as an enabling cause of photosynthesis: it makes the cell able to convert light, water and $\mathrm{CO}_{2}$ into sugar and $\mathrm{O}_{2}$ : something it could not do at all before, due to the absence of certain parts and processes.

Now, minimal causes are not enabling causes of action. In paradigm cases of signals, the state or structure being sent does not provide part of the mechanism for performing the behavior that the signal is designed to produce. In other words, using the causal graph described above, $\mathrm{S}$ is not a minimal cause if setting $\mathrm{S}$ to $\mathrm{S}_{\mathrm{o}}$ leaves the receiver unable to perform $\mathrm{A}_{1}$. As I suggested above, the key intuition behind the notion of a 'minimal cause' is that the intrinsic properties of the state fail to explain behavior, and providing important components of the mechanism for the receiver to be able to act is an extreme way to fail to meet this condition. Of course, we should note that, just as 'being able' comes in degrees, 'being an enabling cause' does as well. Similarly, I take it that whether something qualifies as a signal is a matter of degree. ${ }^{12}$

I think this condition is especially useful for addressing the previous counterexamples. Pollen is not a minimal cause, partly because the genetic material that it carries provides the receiver with the ability to behave in the appropriate way. The genes contained in the pollen are a crucial component of the mechanism required for the receiver to be able to produce its own output (a viable

12 This suggests that the notion of a 'signal' is vague, in the sense that it has borderline cases. In general, I think we should expect that to be true for most biological categories (Godfrey-Smith, 2009). 
seed or plant), so the receiver lacks a complete or nearly complete mechanism for producing the right kind of outcome. In other words, the absence of the intermediate state removes the receiving plant's ability (in the sense specified above) to produce a new plant. Hence pollen blatantly fails to satisfy condition (2) of NEC and, accordingly, should not qualify as a signal. In contrast, molecules employed in plant communication characteristically comply with both conditions of NEC because the receiving plant was already able to produce the response. VOCs such as systemin bind to the plant's receptors and trigger the transcription of various factors, but the components of the mechanisms that produce the defensive response (such as the accumulation of pretoinase inhibitors or ethylene biosynthesis) were already in place in the receiving plant before it sensed systemin. VOCs are not enabling causes because the receiver already possesses the mechanism underlying the output before the signaling molecule is sent. Here molecules just induce the right behavior in the right circumstances, rather than enabling the receiver to behave. ${ }^{13}$

Likewise, this notion makes it possible to appropriately distinguish tool transfer from genuine communication among chimpanzees. Delivering the tool is what enables the receiver to fish termites, so it is not a minimal cause and, accordingly, fails to satisfy a central requirement for signals. In contrast, alarm calls elicit a response that hearers were able to perform before the signal. This condition also provides a way to exclude saliva, since this substance significantly contributes to the mechanism of digestion. Saliva contains certain enzymes that are very important for digesting dietary starches and fat. It does not just trigger the right behavior, but is part of the mechanism that enables the receiver to act in certain ways. Consequently, saliva is not a minimal cause and, as a result, fails to qualify as a signal.

\subsection{Sufficient conditions?}

So far, I have suggested that signals must be minimal causes, and I have argued that a necessary condition for a state to qualify as a minimal cause is that it is a non-enabling cause of behavior. Are these conditions also sufficient for a state to count as a genuine signal? My tentative answer is that being a minimal cause

13 As a reviewer suggested, the notion of a 'nearly complete' mechanism plays an important role here. I explained above some aspects that are relevant for understanding this notion (e.g. it comes in degrees, some aspects might be more important than others, ...), and I hope that future work will allow me to specify this notion in more detail. 
(together with the other conditions included in the Received View) probably suffices for being a signal. It seems to me that any act or structure that is a minimal cause and satisfies the conditions of the Received View plays the role of a genuine signal, as argued above. However, as stated in NEC, being a non-enabling cause is not sufficient for being a minimal cause. Let me elaborate on this last point.

As I suggested above, the key intuition I tried to capture with the notion of a 'minimal cause' is that the intrinsic properties of the signal fail to explain behavior. Being an enabling cause is an extreme way of not satisfying this requirement: if the signal provides part of the mechanism that enables the receiver to act, then the intrinsic properties of the signal obviously play a very important role in explaining the receiver's behavior. However, in other cases the intrinsic properties might be somehow relevant for explaining behavior, even if their contribution does not easily fit into the category of enabling causes. Indeed, I think this intuition is often present in empirical research, even if it is rarely made explicit. To illustrate this idea, let me briefly discuss two examples: the habituation-dishabituation experimental paradigm and the debate between informationalists and manipulationists.

Struhsaker (1967) had observed that vervet monkeys produce a call when they detect a large predator, which causes conspecifics to climb a nearby tree. This, however, was considered insufficient to establish that these calls were signals (Smith, 1991). To provide further evidence, Cheney and Seyfarth performed playback experiments (as discussed above) and also employed the habituation/dishabituation technique. The reasoning behind this test is nicely described by Macedonia and Evans (1993: 181) in the following quote:

If vervet alarm calls are indeed referential then we might expect that the response evoked by playbacks would be mediated principally by the external referent of the call presented (i.e. by the environmental events normally associated with it) rather than simply by acoustic morphology, as in the case of more arbitrary stimuli.

One procedure that allows us to test this prediction runs as follows. Habituation is the phenomenon of reducing one's response to a stimulus through repeated exposure, and dishabituation is the phenomenon of increasing one's response to a new stimulus. Now, suppose that the acoustic properties of calls $\mathrm{X}$ and $\mathrm{Y}$ are more 
similar to each other than the acoustic properties of either $\mathrm{X}$ or $\mathrm{Y}$ are to those of $\mathrm{Z}$ but, nonetheless, $\mathrm{X}$ and $\mathrm{Z}$ are usually produced in similar circumstances $\mathrm{C}$, whereas $\mathrm{Y}$ is elicited in some other circumstances $\mathrm{C}^{*}$. In the experiment, an organism is habituated to $\mathrm{X}$, and then a new stimulus (either $\mathrm{Y}$ or $\mathrm{Z}$ ) is presented. Will the individual that is habituated to $\mathrm{X}$ transfer its reaction to $\mathrm{Y}$ (which is more similar acoustically) or to $\mathrm{Z}$ (which correlates with the same stimulus)? If the habituation is transferred to $\mathrm{Z}$, then this is taken as evidence for its being a signal (Cheney and Seyfarth, 1988; Cheney and Seyfarth, 1990). This experimental design suggests that the status of being a signal goes hand in hand with the acoustic (formal) properties of the state being largely irrelevant (for similar experiments, see Zuberbühler et al., 1999). ${ }^{14}$ A state is not a paradigmatic signal to the extent to which the intrinsic properties of the vehicle (in this case, its formal similarity to another signal) drive action.

I suspect that a similar rationale lies behind recent attempts to question the idea that animal communication involves a transfer of information. ${ }^{15}$ Rendall et al. (2009) have defended the hypothesis first put forward by Dawkins and Krebs (1978), which holds that in many cases where scientists tend to attribute meaningful signals there is actually no information, but only manipulation (see Stegmann, 2013). Their approach is sometimes interpreted as challenging the idea that signaling involves cooperation, but this cannot be the whole story, since they point out that often an informational perspective is inadequate even if there is partial common interest (Rendall and Owren, 2013; Ryan, 2013: 240). So why do they think that an interpretation in terms of signals carrying semantic information is wrong? One suggestion is that they are partly relying on the idea that the best explanation of behavior appeals to the attributes of the signal, rather than its relation to something else (the content). In other words, one way of understanding their challenge is that they reject an interpretation in terms of meaningful signals because these states are not minimal causes.

This suggestion can be illustrated with a recurrent example: the mating call of túngara frogs (Physalaemus pustulosus). Standard approaches to signaling classify the whines and chucks produced by male túngara frogs in mating as signals

14 These experiments are also used to draw conclusions about the cognitive mechanisms involved in signal perception (i.e. to investigate whether it involves cognitive representations or not; see e.g. Kalkman, 2017; Zuberbühler, 1999).

15 I will interpret 'information' as 'semantic information' (i.e. semantic content), not as Shannon information. 
carrying information about body size (larger frogs tend to produce lowerfrequency chucks and send them more often). Rendall et al. (2009) challenge this view by suggesting that the evolution of this phenomenon has been driven by the properties of the signal itself, rather than by its informational content: lowfrequency chucks tend to stimulate an organ, the basiliar papilla, that túngara frogs already possessed before chucks evolved. Thus, in the evolutionary past the ancestors of túngara frogs developed a sensitivity to chucks for some reason that probably has nothing to do with mating and, at some point, male frogs learned how to exploit this sensitivity to attract females. Accordingly, what ultimately drove the evolution of the chuck-signal is the exploitation of the female's perceptual bias by means of the sound properties of chucks. Thus, these authors attempt to challenge the standard informational perspective by stressing the importance of the intrinsic properties of the signal. ${ }^{16}$ To the extent that the form of the vehicle exploits a sensory bias, the intrinsic features of the signal explain behavior. As Ryan (2013: 235) suggests:

The first narrative [informational] about sexual selection in túngara frogs emphasizes how information is encoded and decoded. The second [manipulationist] emphasizes how the structure and influence of signals is tied to morphology and sensory biology.

The view defended in this essay can explain why one of the key strategies for challenging the Received View has been to show that signal evolution is driven by its formal properties rather than by its informational content. ${ }^{17}$ To the extent that intrinsic, non-relational properties of the signal explain behavior, these states diverge from paradigm cases of signaling. ${ }^{18}$

16 An additional reason for thinking that this analysis challenges the traditional attribution of informational content to the túngara's frog's signals is that, on this analysis, it is not obvious that the receiver has a signal-specific response. In any event, my goal here is to provide one reason why the manipulationist framework is in tension with an informational one, but this is compatible with other ways of framing the debate. I would like to thank a reviewer for pressing me on this issue.

17 It should be mentioned that there are certain formal properties of the signal that can explain behaviour without compromising its status as a minimal cause. For instance, when complex signals exhibit some form of systematicity or compositionality, the action might depend on some formal properties. I hope the discussion in the main text illustrates the kind of intrinsic properties that are in tension with the state's being a minimal cause (e.g. its being an enabling cause).

18 Interestingly, those who endorse a representational/informational paradigm reply that their approach enables theorists to apply the same mathematical models across taxa and modalities, whereas manipulationists hold that this position has contributed to neglecting the study of signal design (Seyfartyh et la. 2010; Rendall et al. 2009; Stegmann, 2013: 21). This is precisely what we should expect if we assume that signals are minimal causes: to the extent that a signal is representational, its format is much less explanatory. For this reason, a 
Notice that in the two debates we just considered, the arguments for thinking that some states are not signals did not proceed via the suggestion that they were enabling causes. Failing to pass the habituation/dishabituation test does not imply that the signal is an enabling cause, but it does mean that the response is mainly driven by the formal properties of the vehicle rather than by its content, so it is a way of failing to be a minimal cause. Similarly, manipulatonists do not seem to be arguing that signals enable receivers to act, but rather maintain that the evolution of the sender-receiver configuration is mainly driven by the intrinsic properties of the signal. Consequently, these examples suggest that being an enabling cause is just one way of failing to be a minimal cause and, at the same time, they vindicate the idea that being a minimal cause is a necessary condition for qualifying as a genuine signal.

\section{Connections}

In this final section I would like to briefly consider two additional interesting consequences of Minimalism. The first one concerns the notion of arbitrariness, which has traditionally been considered a distinctive feature of signals (Saussure, 1916). Despite its intuitive support, it is unlikely that signals can be defined by appealing to this concept. The very notion of 'arbitrary' can be specified in many ways, and on any plausible interpretation it seems to be too narrow or too (Stegmann, 2004; Planer and Kalkman, 2019). On the one hand, some signals such as 'indices of quality' are assumed to be relatively non-arbitrary and yet they are usually classified as genuine signals (Maynard-Smith and Harper, 2003; Kalkman, 2019). On the other hand, on many ways of defining this notion, it follows that the relation between many kinds of entities (not only signals) counts as arbitrary. For instance, it is not uncommon to suggest that a state $\mathrm{S}$ is arbitrary with respect to an effect $\mathrm{E}$ to the extent that alternative structures produce $\mathrm{E}$ in close possible worlds where $\mathrm{S}$ is absent. This scheme is exemplified, for instance, in cases of causal preemption, i.e. situations in which $\mathrm{C}$ is the actual cause of $\mathrm{E}$, but there are multiple causal routes that would have led to the same outcome $\mathrm{E}$ if $\mathrm{C}$ had not been the case. It would be absurd to claim that typical cases of causal preemption actually involve signals.

representational/informational perspective facilitates a more widespread use of similar models and tends to pay less attention to the signal's formal properties. 
Now, although signals probably cannot be defined by appealing to arbitrariness, Minimalism can explain why there seems to be a close connection between these two concepts. If Minimalism is right, signals are minimal causes, so the intrinsic properties of the vehicle do not play an important role in explaining behavior. As a result, one should expect there to be alternative vehicles that could easily have played the same causal role. Consequently, the relationship between signals and their effects tends to be relatively arbitrary (in roughly the sense defined above; see also Planer and Kalkman, forthcoming). In contrast, if a state is not a minimal cause, then its intrinsic properties are much more relevant, in which case there will probably be a limited range of alternative states that could have led to similar results. Therefore, the special causal role of signals explains why they tend to be arbitrary (and, hence, why so many people have posited a close relationship between signaling and arbitrariness). I think this is an additional virtue of Minimalism: it accommodates the widespread idea that signals are arbitrary, without endorsing the implausible view that they can be defined as such.

Minimalism also helps to explain some of the heuristics that are used for identifying signals. For instance, why are sounds, color patches or light patterns often hypothesized to have a communicative function? Minimalism suggests an answer: because they cause adaptive behavior while failing to provide the mechanism for action. Typically sounds, color patches and light patterns trigger action without enabling receivers to act, so if they produce a certain behavior, a plausible explanation is that they carry semantic information about something else. Thus, Minimalism predicts that in actual scientific (and non-scientific) practice, discovering an act or structure to be a minimal cause will be taken as evidence for its being a signal.

Butlin (forthcoming) has recently made a very interesting contribution that bears some similarities to Minimalism. Like the present account, Butlin aims to solve the liberality problem for teleological theories (although he focuses on intra-, rather than inter-organismic signaling), and he also suggests an additional condition. According to him, receivers of signals are typically 'capable of independent action' (p.1), by which he means that receivers

have at least two separate components, working with a degree of independence from one another. One of these components must have the function which includes activating further changes conditional on the occurrence of the representation, and 
the other must be responsible for generating the behavior to which the representation contributes. (p.12)

While there are some obvious similarities between our suggestions (and lacking the space for a more detailed analysis), I would like to briefly highlight some important differences between my account and his. On the one hand, my proposal seeks to identify a distinctive causal property of signals, namely their being minimal causes. Being an enabling cause is one way of failing to be a minimal cause but, as I pointed out in section 4.3, in the discussion of the habituation/dishabituation paradigm and the informational/manipulationist debates, there might be other ways of failing to be a minimal cause. In contrast, Butlin defines his additional condition in terms of a property of receivers, rather than in terms of the causal profile of the signal: his main goal is to explore the idea of an active (rather than a passive) consumer. Secondly, his condition requires that the receiver contains two separate mechanisms, one registering the signal and another being responsible for an active response. This is not required by Minimalism. As a consequence, whereas Butlin's proposal is supposed to exclude so-called 'receptors' (states that are triggered by specific stimuli and cause a particular behavior downstream), in principle this is not excluded by my account. Furthermore, Butlin seeks to provide a sufficient condition for qualifying as a genuine representation, whereas I put forward a necessary condition, which is included in a set of jointly sufficient conditions for a state to qualify as a signal (see section 4.3.). Finally, notice that my account is compatible with Butlin's proposal, so one should not regard them as rivals between which we must choose, but rather as different strategies for addressing the liberality objection. One could coherently embrace one of them, both or neither.

Finally, I would like to briefly consider a potential worry. Imagine that we could come up with a very special situation in which a signal happens to also be an enabling cause of some behavior. I think there might be different strategies for accommodating this sort of case, but notice that Minimalism holds that, as a signal, an act or structure is a minimal cause. Thus, this approach is compatible with the same act or structure playing other causal roles on some occasions. In other words, Minimalism defines the role that a particular state is supposed to play as a signal, and this is consistent with that state having other effects in some specific contexts. 


\section{Conclusion}

Wrapping up, I have argued that the Received View of communication is a powerful framework that needs to be supplemented with an additional condition: signals are minimal causes. I have tried to clarify this notion by specifying two necessary conditions for a state to qualify as a minimal cause, namely being a cause, but not an enabling one. I think this is a principled addition that, furthermore, gives the right results in the context of animal communication and fits the explanatory role that signals are supposed to play. Additionally, this view draws some illuminating connections between conceptual considerations and empirical research, and provides new insights into the informational/manipulationist debate as well as into the arbitrariness of representations. In any case, I hope this paper has at least contributed to highlighting the fact that signals have a special causal role. Fully exploring the notion of a 'minimal cause' and its consequences remains work for future research.

ACKNOWLEDGMENTS: I would like to thank Leonardo Bich, Mihnea Capraru, Marta Campdelacreu, Sabrina Engesser, Samir Okasha, Javier González de Prado Salas, Kirsty Graham, John Horden, Manolo Martínez, Richard Moore, Saúl Pérez, Aida Roige, Miguel Ángel Sebastián, Nicholas Shea, Dan Zeman and the audiences at the VII SEFA Conference, the Seminario de Filosofía de la Mente at the Instituto de Investigaciones Filosóficas at the Universidad Nacional Autónoma de México, the ISCHPSSB 2019 Conference at the University of Oslo and the 1st Varieties of Information Workshop on Animal Communication at the University of Barcelona. Financial support was provided by the Ministerio de Economía y Competitivdad through the projects 'La Complejidad de la Percepción: un enfoque multidimensional' (FFI2014-51811-P) and 'Varieties of Information' (PGC2018-101425-B-I00).

\section{References}

Abrams, M. (2005) Teleosemantics Without Natural Selection. Biology and Philosophy. 20(1): 97-116

Ackerman, J. D. (2000) Abiotic pollen and pollination: Ecological, functional, and evolutionary perspectives. Plant Systematics and Evolution. 222(1-4)167-185

Allen, C. and M. Bekoff (1997) Species of Mind. MIT Press

Artiga, M. (2014) Signaling Without Cooperation. Biology and Philosophy. 29: 357-378. 
Baluska, F., S. Mancuso and S. Volkmann (eds.) (2006) Communication in Plants. Springer

Baluska, F., V. Ninkovic (eds.) (2006) Plant Communication form an Ecological Perspective. Springer

Bradbury, J. and S. Vehrencamp (1998) Principles of Animal Communication. Sinauer Associates

Butlin, P. (forthcoming) Representation and the Active Consumer. Synthese

Calcott, B., P. Griffiths, A. Pocheville (forthcoming) Signals that Make a Difference. British Journal for the Philosophy of Science.

Cheney, D. and R. Seyfarth (1988) Assessment of meaning and the detection of unreliable signals by vervet monkeys, Animal Behavior, 36(2): 477-486

Cheney, D. and R. Seyfarth (1990) How Monkeys see the World. University of Chicago Press

Cheney, D. and R. Seyfarth (2007) Baboon Metaphysics. University of Chicago Press

Corrado, G., R. Sasso, M. Pasquariello, L. Iodice, A. Carretta, P. Cascone, L. Ariati, M. C. Diglio, E. Guerrieri and R. Rao (2007) Systemin Regulates Both Systemic and Volatile Signaling in Tomato Plants. Journal of Chemical Ecology. 33:669-681

Coppola, M., P. Cascone, V. Madonna, I. Di Lelio, F. Esposito, C. Avitabile, A. Romanelli, E. Guerrieri, A. Vitiello, F. Pennacchio, R. Rao and G. Corrado (2017) Plant-to-plant communication triggered by systemin primes anti-herbivore resistance in tomato. Scientific Reports 7, 15522

Dawkins R. and J. Krebs (1978) Animal Signals: information or manipulation. In J. Krebs \& Davies (eds.) Behavioral Ecology: An Evolutionary Approach. Blackwell 
Scientific.

De Waal, F. (1982) Chimpanzee Politics: Power and Sex Among Apes. John Hopkins University

Diggle, S. et al. (2007) Evolutionary Theory of bacterial quorum sensing: when a signal not a signal? Philosophical Transactions of the Royal Society B, 1-9

Evans Ch. and L. Evans (1999) Chicken food calls are functionally referential. Animal Behavior. 58(2):307-319.

Evans Ch. and L. Evans (2007) Representational Signalling in Birds. Biology Letters. 3(1): 8-11

Frick, R., Bich, L. and Moreno, A. (2019) An organisational approach to biological communication. Acta Biotheoretica, 67(2), 103-128

Friedman J. and S. Barrett (2009) Wind of change: new insights on the ecology and evolution of pollination and mating in wind-pollinated plants. Annals of Botany. 103(9): 1515-1527.

Garson, J. (2013) The Functional Sense of Mechanism. Philosophy of Science. 8o(3): 317-333

Glennan, S.S. (2002) Rethinking Mechanistic Explanation. Philosophy of Science, 64: 605-626

Glennan, S.S. (2017) New Mechanical Philosophy, Oxford University Press.

Godfrey-Smith, P. (2009) Darwinian Populations and Natural Selection. OUP

Godfrey-Smith, P. (2013) Information and Influence in Sender-Receiver Models. In Stegmann, U. (ed.) Animal Communication Theory. Information and Influence. Cambridge University Press, 2013.

Godfrey-Smith, P. and M. Martinez (2013) Communication and Common Interest. PLOS, 9(11) 
Hasson, O. (1994) Cheating Signals. Journal of Theoretical Biology, 167(3), 223238

Kalkman, D. (2017) Information, Influence, and the Causal-explanatory Role of Content in Understanding Receiver Responses. Biology and Philosophy, 32(6): 124

Kalkman, D. (2019) New Problems for Defining Animal Communication in Informational Terms. Synthese, 196(8): 3319-3336

Karakashian, S. J., Gyger, M., and Marler, P. (1988) Audience effects on alarm calling in chickens (Gallus gallus). Journal of Comparative Psychology, 102(2), 129-135.

Machamer, P.K., L. Darden, and C.F. Craver (2000) Thinking about Mechanisms. Philosophy of Science, 67: 1-25

Macedonia J. and Ch. Evans (1993) Variation among Mammalian Alarm Call Systems and the Problem of Meaning in Animal Signals. Ethology, 93: 177-197

Maier, J. (2014) Abilities. In E. Zalta, Stanford Encyclopedia of Philosophy, url: <https://plato.stanford.edu/archives/spr2018/entries/abilities/>

Manser, M., Bell M. Fletcher (2001) The information that receivers extract from alarm calls in suricates. Proceedings of the Royal Society B: Biological Sciences. 268(1484):2485-91.

Maynard-Smith, J. and D. Harper (2003) Animal Signals. OUP

Millikan, R. (1984) Language, Thought and Other Biological Categories. MIT Press

Musgrave, S., D. Morgan, E. Lonsdorf, R. Mundry and C. Sanz (2016) Tool transfers are a form of teaching among chimpanzees. Scientific Reports 6, 34783

Neander, K. (2012) Teleological Theories of Mental Content. In E. Zalta, Stanford 
url: <https://plato.stanford.edu/archives/spr2018/entries/content-teleological/>

Otte, (1974) Effects and Functions in the Evolution of Signaling Systems. Annual Review of Ecology and Systematics. 5: 385-417

Papineau, D. and G. McDonald (2006) Teleosemantics. OUP

Planer, R. and D. Kalkman (forthcoming) Arbitrary Signals and Cognitive Complexity. British Journal for the Philosophy of Science. 78: 233-240

Rendall, D., M. Owren and M. Ryan (2009) What do animal signals mean? Animal Behavior. 78: 233-240

Rendall D, and M. Owren (2013) Communication without meaning or information: abandoning language-based and informational constructs in animal communication theory. In U. Stegmann (ed.) Animal communication theory: information and influence. Cambridge University Press, 151-182

Rogers, L., L. Stewart, and G. Kaplan (2018) Food Calls in Common Marmosets, Callithrix jacchus, and Evidence That One Is Functionally Referential. Animals, 8(7): 99

Ryan, C. (2000) The systemin signaling pathway: differential activation of plant defensive genes. Biopchimica et Biophysica Acta. 1477:112-121

Ryan, M. (2013) The Importance of Integrative Biology to Sexual Selection and Communication. In U. Stegmann (ed.) Animal communication theory: information and influence. Cambridge University Press, 233-256

Saussure, F. (1916) Cours de linguistique générale. Payot.

Scarantino, A. (2013a) Animal communication as information-mediated influence. In U. Stegmann (ed.) Animal communication theory: information and influence. Cambridge University Press, 63-88

Scarantino, A. (2013b) Rethinking Functional Reference. Philosophy of Science, 
80(5): 1006-1018

Scarantino, A., and Clay, Z. (2015) Contextually variable signals can be functionally referential. Animal Behaviour, 100, e1-e8

Schorkopf, D. L., S. Jarau and W. Francke (2007) Spitting out information: Trigona bees deposit saliva to signal resource locations. Proceedings of the Royal Society: Biological Sciences,274(1611): 895-8

Scott-Phillips, T. C. (2008) Defining Biological Information. Journal of Evolutionary Biology, 21: 387-395

Searcy, W. and S. Novicky (2005) The Evolution of Animal Communication. Princeton University Press

Seyfarth, R., D Cheney and P. Marler (1980) Monkey responses to three different alarm calls: evidence of predator classification and semantic communication. Science, 210 (4471): 801-3

Seyfarth, R, D. Cheney, Th. Bergmann, J. Fischer, K. Zuberbühler and K. Hammerschmidt (2010) The Central Importance of Information in Studies of Animal Communication. Animal Behavior 80(1): 3-8

Seyfarth, R. and D Cheney (2017) The Origin of Meaning in Animal Signals. Animal Behavior, 124: 339-346

Shea, N. (2018) Representations in Cognitive Science. Oxford University Press

Shettleworth, S. (2010) Cognition, Evolution and Behavior. Oxford University Press

Smith, W. J. (1991) Animal Communication and the Study of Cognition. In Carolyn A. Ristau, and Donald R. Griffin (eds.). Cognitive Ethology: The Minds of Animals: Essays in Honor of Donald R. Griffin, 209-230. Hillsdale, N.J: L. Erlbaum Associates.

Stegmann, U. (2004) The Abritrariness of the Genetic Code. Biology and 
Philosophy 19 (2): 205-222

Stegmann, U. (2009) A Consumer-Based Teleosemantics for Animal Signals. Philosophy of Science, 76: 864-875.

Stegmann, U. (2013) Animal Communication Theory: Information and Influence. Cambridge University Press

Sterelny, K. (1995) Basic Minds. Philosophical Perspectives. 9: 251-70

Struhsaker, T.T. (1967) Auditory communication among vervet monkeys (Cercopithecus aethiops). In Stuart A. Altmann (editor) Social communication among primates. Chicago: University of Chicago Press, pp. 281-324.

Ueda, H., Y. Kikuta and K. Matsuda (2012) Plant Communication. Plant Signaling and Behavior. 7(2): 222-226

Wang, L., E. Einig, M. Almeida-Trapp, M. Albert, J. Fliegmann, A. Mithöfer, H. Kalbacher and G. Felix (2018) The Systemin receptor SYR1 enhances resistence of tomato against herbivorous insects. Nature Plants, 4: 152-156

Wheeler, B., and J. Fisher (2012) Functionally referential signals: A promising paradigm whose time has passed. Evolutionary Anthropology 21(5)

Wheeler, B., and J. Fisher (2015) The blurred boundaries of functional reference: a response to Scarantino \& Clay. Animal Behaviour, 100, e9-e13

Wiley, R. H. (1994) Errors, Exaggeration, and Deception in Animal Communication. In L. Real, Behavioral Mechanisms in Ecology, University of Chicago Press, University of Chicago, ch. 7, 157-189.

Wiley, R. (2013) Communication as a transfer of information: measurement, mechanism and meaning. In Stegmann, U. (ed.) Animal Communication Theory. Information and Influence. Cambridge University Press, 2013.

Wilson, E. (1975) Sociobiology: the New Synthesis. Harvard University Press 
Woodward, J. (2003) Making Things Happen: A Theory of Causal Explanation. New York: Oxford University Press.

Woodward, J. (2010) Causality in Biology: stability, specificity and the choice of levels of explanation. Biology and Philosophy. 25: 287-318

Zahavi A, Zahavi A (1997) The handicap principle: a missing piece of Darwin's puzzle. Oxford University Press

Zuberbühler, K. (1999) Referential labelling in Diana monkeys. Animal Behavior, 59(5):917-927

Zuberbühler, K. (2000) Predator-Specific Alarm Calls in Campbell's Monkeys, Cercopithecus campbelli. Behavioral Ecology and Sociobiology, 50(5): 414-422

Zuberbühler, K., D. Cheney and R. Seyfarth (1999) Conceptual Semantics in a Nonhuman Primate. Journal of Comparative Psychology, 113(1): 33-42 